Ann. Génét. Sél. anim., I980, 12 (3), 267-280.

\title{
Etude comparative des performances de reproduction des truies de races Créole et Large white en Guadeloupe
}

\author{
I. CANOPE et Y. RAYNAUD( $\left.{ }^{1}\right)$
}

avec la collaboration technique de F. HEDREVILI,E, E. DESPOIS, B. Racon

Station de Recherches Zootechniques,

I.N.R.A., Antilles-Guyane,

Domaine Duclos, 97 I70 Petit-Bourg

\section{Résumé}

La population porcine des Antilles françaises est représentée principalement par deux types génétiques : le Large White et le porc indigène appelé "porc Créole " ou porc "Planche ".

A partir des enregistrements de notre troupeau expérimental, les performances de reproduction de ces deux souches ont été comparées.

L'âge et le poids à la puberté sont respectivement de $\mathrm{r} \mathrm{r}, 4$ jours et $52 \mathrm{~kg}$ pour les Créoles et 275 jours et $107 \mathrm{~kg}$ pour les Large White.

Les autres caractéristiques comme l'âge et le poids à la saillie fécondante, l'intervalle entre le sevrage et l'œstrus suivant l'intervalle entre le sevrage et la saillie fécondante, la taille de la portée à la naissance, le poids moyen de la portée à la naissance, la taille de la portée au sevrage, le poids de la portée au sevrage sont respectivement de $2 \pi 2,9$ jours, $53,8 \mathrm{~kg} ; 22,96$ jours, 28,47 jours, 7,33; 906; 6,7I et 5322 pour les Créoles et 283,0; r I0,o kg, 24,6; 36,2; 9,43; I $377 \mathrm{~g} ; 7,42 ; 8$ o30 pour les Large White. Une influence de la saison sur les performances a été observée. sèche.

Dans nos conditions climatiques les meilleurs résultats sont obtenus au cours de la saison portée.

En ce qui concerne la croissance des porcelets elle varie en sens inverse de la taille de la

Enfin le calcul de la productivité numérique donne les chiffres suivants : 13,82 pourl es Créoles et $\mathbf{r}_{4}, 6$ pour les Large White.

\section{I. - Introduction historique}

La population porcine de la Guadeloupe est constituée essentiellement de deux sous-ensembles d'importance inégale : le porc Large white, race pure d'introduction récente (une vingtaine d'années environ) et la population locale plus

(1) Ingénieur agronome de l'E.N.S.A. de Toulouse (Volontaire Aide Technique en Guadeloupe). 
connue sous le nom de porc Créole ou porc "Planche". Ces porcs locaux seraient le résultat de croisements entre des animaux de type ibérique introduits dès le $\mathrm{XVI}$ e siècle et des porcs français dont l'introduction date de la colonisation des Antilles à partir de I635 (DUTERTRE I667-I67I, LABAT (I822).

Par la suite cette population de départ s'est trouvée largement métissée au fil des siècles à la suite d'introduction de races anglaises (Large black, Yorkshire), américaines (Duroc, Hampshire...) et françaises (Normand, Craonnais...). Il en résulte un grand polymorphisme qui se manifeste encore de nos jours au niveau de la morphologie, du format et de la coloration (LAUVERGNE et CANOPE, I979).

Les caractéristiques zootechniques du pore Créole dans ses conditions naturelles ont fait 1'objet d'une première estimation dans la monographie de LEMENTEC (1970).

Nous rappelons que cette étude avait souligné le faible niveau des performances d'élevage ( 7 porcelets à la naissance et un poids moyen de $6210 \mathrm{~g}$ à 60 jours) et d'engraissement (42,3 $\mathrm{kg}$ à I2 mois).

Cet article se propose de faire le point sur les performances de reproduction d'un troupeau de race locale en comparaison avec des porcs Large White élevés dans les mêmes conditions.

\section{II. - Materiel et méthodes}

\section{I. - Matériel animal}

Les données analysées proviennent du troupeau expérimental de la station de recherches zootechniques du C.R.A.A.G. (INRA) située en Guadeloupe dans les conditions géographiques et climatiques suivantes :

$\begin{array}{ll}\text { - latitude } & 16^{0} \text { Nord } \\ \text { - longitude } & 6 \mathbf{I}^{0} \text { Ouest } \\ \text { - température moyenne } & \\ \text { annuelle } & 24^{\circ} \mathrm{C} \\ \text { - température minimum } & 20^{0} 5 \\ \text { - température maximum } & 27^{0} 5 \\ \text { - humidité relative } & \\ \begin{array}{l}\text { (minimum) } \\ \text { - humidité relative }\end{array} & 70 \mathrm{p} \text {. Ioo } \\ \text { (maximum) } & 95-\mathrm{Io0} \text { p. Ioo }\end{array}$

Ce troupeau Créole, constitué en I973, comprend actuellement 43 truies et 5 verrats élevés côte à côte et de la même manière que le troupeau de race Large White introduit en I964 (40 truies et 5 verrats). Les effectifs analysés sont cependant variables car les observations n'ont pu être effectuées sur la totalité des animaux.

\section{2. - Conduite de l'élevage}

Les bâtiments d'élevage sont de type semi plein air; seule l'aire de couchage est couverte; les truies en gestation sont logées par groupe de ro et alimentées individuellement. Les cases de lactation sont conçues de manière à permettre la 
distribution de granulés aux porcelets dès 1'âge de ro jours. Le sevrage est réalisé à 35 jours \pm 3 jours.

Le contrôle de l'œstrus des jeunes truies débute dès le poids de $45 \mathrm{~kg}$ (I6o jours) pour les Créoles et à $70 \mathrm{~kg}$ ( 189 jours) pour les Large White à l'aide d'un verrat, mais celles-ci ne sont pas saillies avant d'avoir atteint le poids de 52 et roo $\mathrm{kg}$ respectivement pour les Créoles et les Large White. Après le sevrage, la détection des chaleurs des truies commence au terme d'une diète hydrique de 48 heures.

\section{3. - Alimentation}

Les jeunes truies des deux races sont nourries en semi-ad libitum : la ration journalière est ajustée en fonction de 1'appétit avec un aliment unique (croissancefinition) à base de maïs ayant 15 p. Ioo de matières azotées et 3200 kcal d'énergie digestible par kilo.

Les truies en gestation reçoivent 2 à $2,5 \mathrm{~kg}$ d'aliment par jour; il s'agit d'un aliment à $3200 \mathrm{kcal}$ et I4 p. Ioo de matières azotées.

En cours de lactation elles sont nourries à volonté avec un aliment à $\mathrm{I} 6 \mathrm{p}$. Ioo de matières azotées et $3200 \mathrm{kcal}$. Le libre accès aux abreuvoirs permet une alimentation en eau à volonté.

\section{III. - Résultats}

La puberté est observée à l'âge de $275 \pm 7$ jours (tabl. I) pour les jeunes truies Large White et I7I, 4 jours pour les Créoles à des poids deux fois plus faibles pour ces dernières $(52,3 \pm 2,0$ contre $107 \pm 2,7$ pour les Large White). La relation existant entre le poids à la puberté et le poids adulte de la jeune truie n'a pu cependant être précisée, les animaux n'ayant pas été pesés lors de leur première mise bas.

- L'intervalle séparant la première saillie de la fécondation chez les truies, qui dépend du nombre de retours en chaleur ne diffère pas significativement d'un type génétique à un autre (tabl. I).

En revanche, l'intervalle qui sépare l'œestrus et la fécondation après le sevrage est deux fois plus grand (I2 jours) chez la truie Large White (tab1. 2).

- La durée de gestation (tabl. 2) est d'environ II4 jours pour les deux races avec cependant une variabilité légèrement plus grande pour les Créoles quelque soit le numéro de portée (tabl. 4).

Le nombre de tétines fonctionnelles (tab1. 2) est significativement plus élevé en race Large White White qu'en race Créole (I3,4 contre I2,2). I1 s'agit d'un caractère important chez la truie allaitante car il conditionne le nombre de porcelets qu'elle peut nourrir.

Nous avons regroupé dans le tableau 3 les valeurs moyennes de la taille et du poids de la portée aux trois âges habituels de référence de la croissance du porcelet (naissance, 3 semaines, 5 semaines). Les résultats sont dans l'ensemble plus faibles pour les porcelets Créoles que pour les Large White.

Le nombre de porcelets nés vivants est plus élevé chez la truie Large White d'environ $28 \mathrm{p}$. Ioo à la naissance. Mais cette supériorité n'est plus que de II p. Ioo 


\section{TABLEAU I}

Valeurs moyennes des paramètres de reproduction Statistical parameters and significance of difference

\begin{tabular}{|c|c|c|c|c|}
\hline \multicolumn{2}{|c|}{ Types génétiques } & Créole & Large White & $\begin{array}{l}\text { Signification } \\
\text { statistique }(t)\end{array}$ \\
\hline \multirow{4}{*}{ Ages observés (jours) } & à la puberté(1) & $\mathrm{I} 7 \mathrm{I}, 4 \pm 3,54$ & $275 \pm 7,0$ & $\mathrm{I}_{3}, 86(* *)$ \\
\hline & $\begin{array}{l}\text { a paillie } \\
\text { sa }\end{array}$ & $204,6 \pm 7,0$ & $275 \pm 7,0$ & $6, \mathrm{I} 8(* *)$ \\
\hline & fécondante & $212,9 \pm 7,6$ & $283,0 \pm 8,3$ & $5,59\left(^{* *}\right)$ \\
\hline & $\begin{array}{l}\text { à la première } \\
\text { mise-bas }\end{array}$ & $327,2 \pm 7,5$ & $\pm 8,4$ & $5,46(* *)$ \\
\hline \multirow{2}{*}{\multicolumn{2}{|c|}{ Poids $(\mathrm{kg}) . .+.\left\{\begin{array}{l}\text { à la puberté } \\
\begin{array}{l}\text { à la saillie } \\
\text { fécondante }\end{array}\end{array}\right.$}} & $52,3 \pm 2,0$ & $107 \pm 2,7$ & $16,65\left(^{* *}\right)$ \\
\hline & & $53,8 \pm 2,6$ & $\pm 3,27$ & $12,27\left(^{* *}\right)$ \\
\hline
\end{tabular}

(1) Les moyennes ont été calculées sur un échantillon contemporain de 30 créoles et 25 Large white. $(* *)$ Différence hautement significative $(\mathrm{P}<0$,or $)$.

TABLEAU 2

Valeur moyenne des autres paramètres de reproduction Significance of differences between breeds

\begin{tabular}{|c|c|c|c|}
\hline Types génétiques & Créole & Large White & $\begin{array}{l}\text { Signification } \\
\text { statistique } \\
\text { (valeur de } t \text { ) }\end{array}$ \\
\hline Durée de gestation $(\mathrm{j})\left({ }^{1}\right)\left(5^{6-45}\right) . .$. & I I 4, IO $\pm 0,44$ & I I $4,3 \pm 0,2$ I & 0,29 (n.s.) \\
\hline Intervalle sevrage-œstrus $(24-34)$. . . & $22,96 \pm 3,0$ & $24,6 \pm 2,45$ & 0,20 (n.s.) \\
\hline 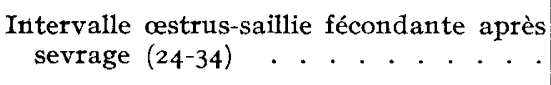 & 6 & I 2 & - \\
\hline 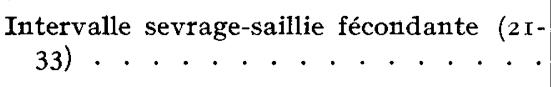 & $28,4^{8} \pm 3,6$ & $3^{6,2} \pm 0,46$ & I, I 7 (n.s.) \\
\hline $\mid \begin{array}{c}\text { mâles } \\
(370-125)\end{array}$ & $\mathrm{I} 2, \mathrm{I} \pm \mathrm{I}, 2$ & $13,2 \pm \mathrm{r}, 32$ & $8,46(* *)$ \\
\hline Nbre de tétines $\cdot\left\{\begin{array}{l}\text { femelles } \\
(35 \mathrm{I}-92)\end{array}\right.$ & $\mathrm{I} 2,2 \pm \mathrm{I}, \mathrm{I}$ & I $3,7 \pm \mathrm{r}, 24$ & I I ,38 $(* *)$ \\
\hline
\end{tabular}

(1) I,es chiffres entre parenthèses correspondent aux effectifs en animaux Créole et Large white respectivement.

$(* *)$ Différence hautement significative $(\mathbf{P}<\mathrm{o,or})$. 


\section{TABLEAU 3}

Taille de la portée et caractéristiques du porcelet Differences between breeds for litter size and weight of piglets

\begin{tabular}{|c|c|c|c|c|}
\hline Paramètres & Types génétiques & Créole & Large White & $\begin{array}{l}\text { Signification } \\
\text { statistique } \\
\text { (valeur de } t \text { ) }\end{array}$ \\
\hline \multirow{3}{*}{ Taille de la portée } & Naissance & $7,33 \pm 0,24$ & $9,43 \pm 0,3^{8}$ & $5,68(* *)$ \\
\hline & 3 semaines & $6,95 \pm 0,24$ & $7,53 \pm 0,23$ & I,7I (n.s.) \\
\hline & $\begin{array}{c}\text { Sevrage } \\
5 \text { semaines }\end{array}$ & $6,71 \pm 0,25$ & $7,42 \pm 0,23$ & $2, \mathrm{I} \quad(*)$ \\
\hline \multirow{3}{*}{ Poids moyen $(\mathrm{g})$} & Naissance & $906 \pm 22,6$ & I $377 \pm 22,8$ & I $4,66(* *)$ \\
\hline & 3 semaines & $3596 \pm 12,1$ & $4970 \pm 109$ & 10,6 (**) \\
\hline & $\begin{array}{c}\text { Sevrage } \\
5 \text { semaines }\end{array}$ & $5322 \pm 200$ & $8030 \pm \mathrm{I} 59$ & $33,93\left(^{* *}\right)$ \\
\hline \multirow{2}{*}{ Pertes $(\%) \cdot \cdot \cdot \cdot$} & $\mathrm{N}-3$ semaines & 5,2 & $20, \mathrm{I}$ & - \\
\hline & $\mathrm{N}-5$ semaines & 8,5 & $2 \mathrm{I}, 3$ & - \\
\hline \multirow{2}{*}{ Sex-ratio } & Mâles & $5 \mathrm{I}, 9$ & $5 \mathrm{I}, \mathrm{O}$ & - \\
\hline & Femelles & $4^{8, I}$ & 49,0 & - \\
\hline
\end{tabular}

(*) Différence significative $(\mathrm{P}<0,05)$.

(**) Différence hautement significative $(\mathrm{P}<\mathrm{o}$,or $)$.

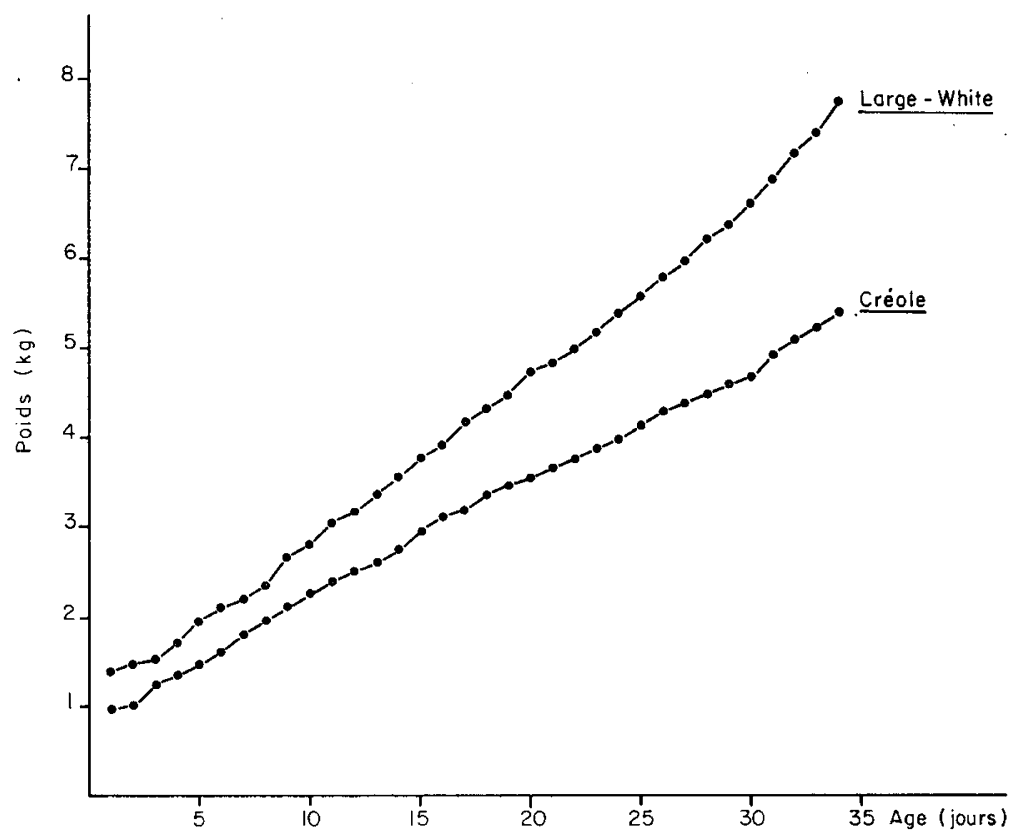

FIG. I. - Croissance comparée de porcelets Large White et Créoles de o à 35 jours

Compared growth of Large White and Creole piglets from o to 35 days 


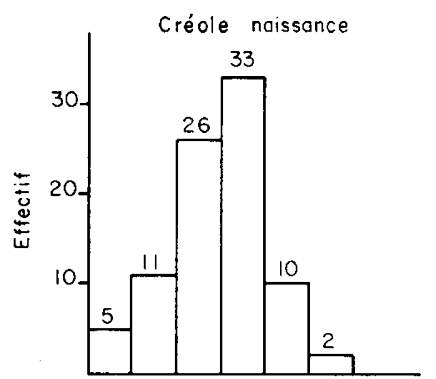

$3-4.5-6 \cdot 7-8 \cdot 9-10.11-12 \cdot 13-14$

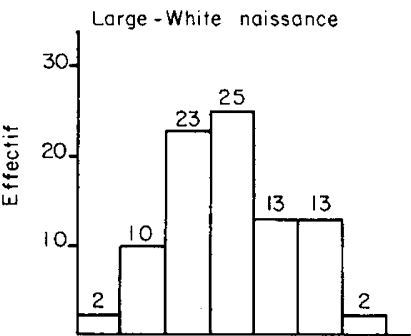

$3-4.5-6.7-8.9-10.11-12 \cdot 13-14.15-16$

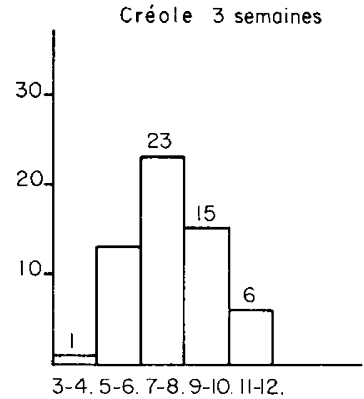

Toille portée

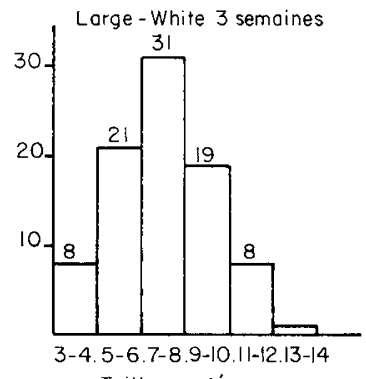

Créole 5 semaines

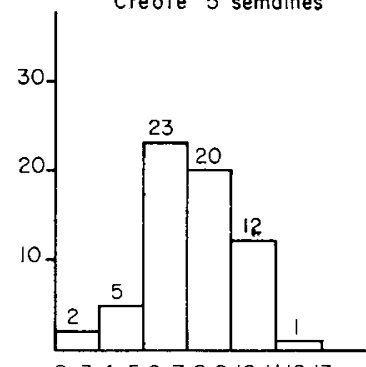

$2-3.4-5.6-7.8-9 \cdot 10-11.12-13$.

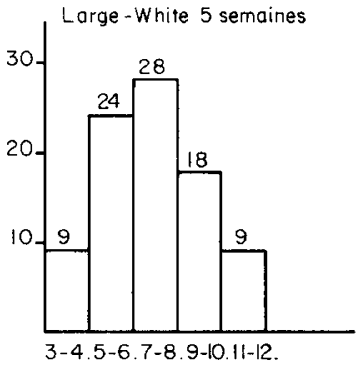

FIG. 2. - Taille de la portée à la naissance à 3 et 5 semaines dans les deux vaces Créole et Lavge White Litter size at birth, 3 and 5 weeks in Creole and Large White

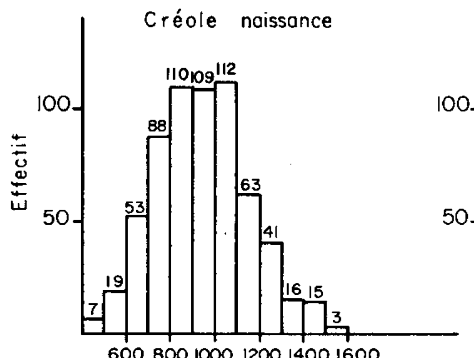

600.800 .1000 .1200 .1400 .1600

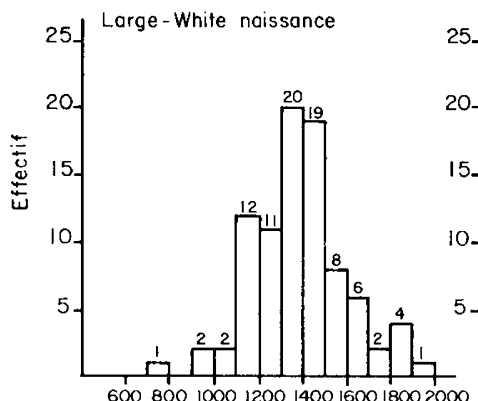

Créole 3 semoines
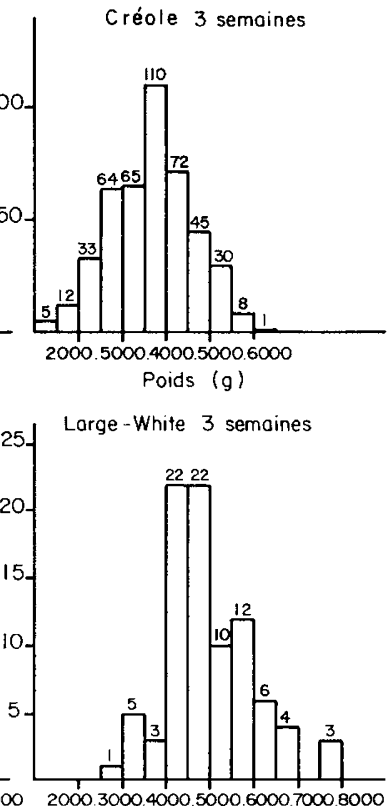
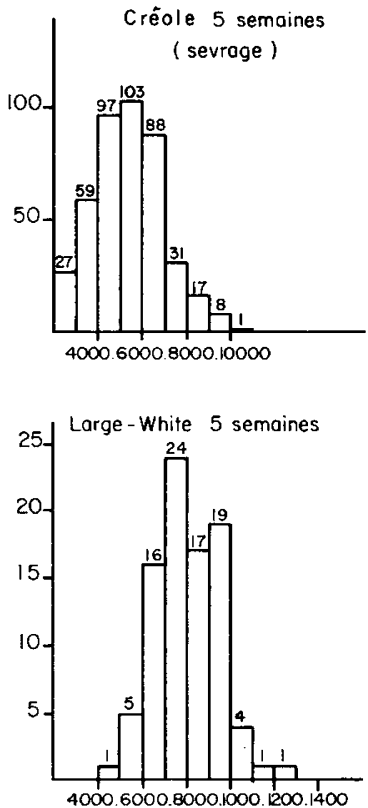

Poids $(g)$

FIG. 3. - Poids des porcelets Créoles et Large White à la naissance, 3 et 5 semaines

Weight of Creole and Large White piglets at birth, 3 and 5 weeks 


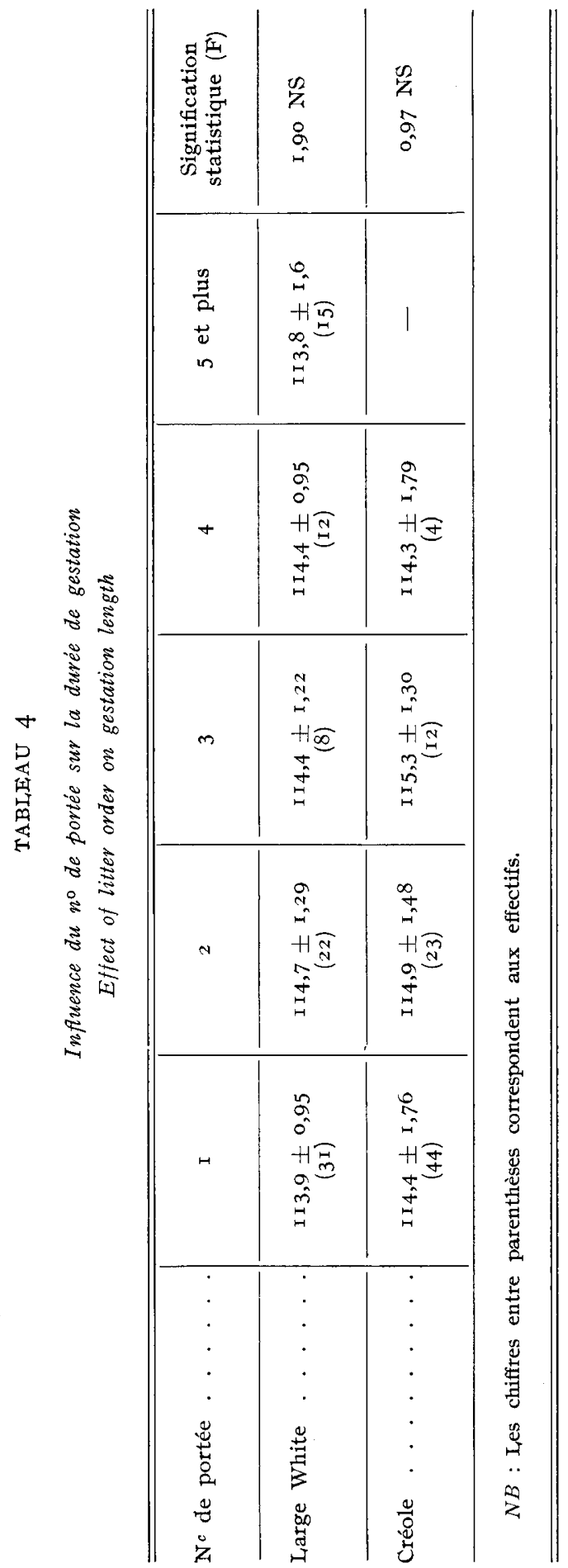


TABLEAU 5

Infuence de la taille de la portée sur le poids des porcelets

Large White et créoles scurés à 5 semaines

Effect of litter size on piglet weight in the two brceds

\begin{tabular}{|c|c|c|c|c|}
\hline $\begin{array}{l}\text { Classe de " taille" } \\
\text { (nombre de } \\
\text { porcelets sevrés) }\end{array}$ & Races $\left({ }^{1}\right)$ & $\begin{array}{l}\text { Poids moyen } \\
\text { au sevrage } \\
\text { (5 semaines) }\end{array}$ & $\begin{array}{l}\text { Gain moyen } \\
\text { quotidien }\end{array}$ & $\begin{array}{c}\text { Pertes } \\
\text { après sevrage }\left({ }^{2}\right)\end{array}$ \\
\hline \multirow{2}{*}{5 et 6} & Créole (I 2) & $7208^{a} \pm 1,8$ & $\mathrm{I}_{72} \pm 48, \mathrm{O}$ & - \\
\hline & Large White (29) & $8310 \pm 0,7$ & $196 \pm 20,0$ & - \\
\hline \multirow{2}{*}{7 et 8} & Créole (32) & $49^{22^{a}} \pm 0,8$ & I I $4 \pm 22,0$ & 3,2 \\
\hline & Large White (30) & $77^{80^{b}} \pm 0,7$ & $\mathrm{I} 86 \pm 19, \mathrm{O}$ & - \\
\hline \multirow{2}{*}{9 et pluss } & Créole (57) & $5^{600^{a}} \pm 0,6$ & $\mathrm{I} 3^{\circ} \pm \mathrm{I} 4,7$ & $\mathrm{I}, 8$ \\
\hline & Large White (1 8) & $7330^{b} \pm \mathrm{I}, 34$ & $169 \pm 35,4$ & 5,6 \\
\hline
\end{tabular}

(1) Le chiffre entre parenthèses correspond aux effectifs des classes.

(2) Par rapport aux sevrés à 5 semaines (jusqu'à 8 semaines).

$(a, b)$ : Moyennes significativement différentes.

au sevrage sans doute en raison de la rusticité et des qualités maternelles de la truie Créole.

Le taux de masculinité (tabl. 3) toutes portées confondues est d'environ 52 p. roo pour les deux races.

De la naissance au sevrage, la croissance des porcelets est supérieure chez les Large White (fig. I); l'écart en poids $(27,4$ p. roo) constaté à la naissance se retrouve pratiquement au sevrage $(28,5 \mathrm{p}$. IOo).

Les histogrammes correspondant à la taille de la portée et au poids aux différents âges (naissance, 3 semaines, 5 semaines) sont présentés dans les figures 2 et 3 .

La croissance est, en général d'autant meilleure que le nombre de porcelets est plus faible (tab1. 5).

Les variations de la taille de la portée en fonction du numéro de portée figurent au tableau 6 .

Enfin, les influences saisonnières sur les performances d'élevage figurent au tableau 7 . 


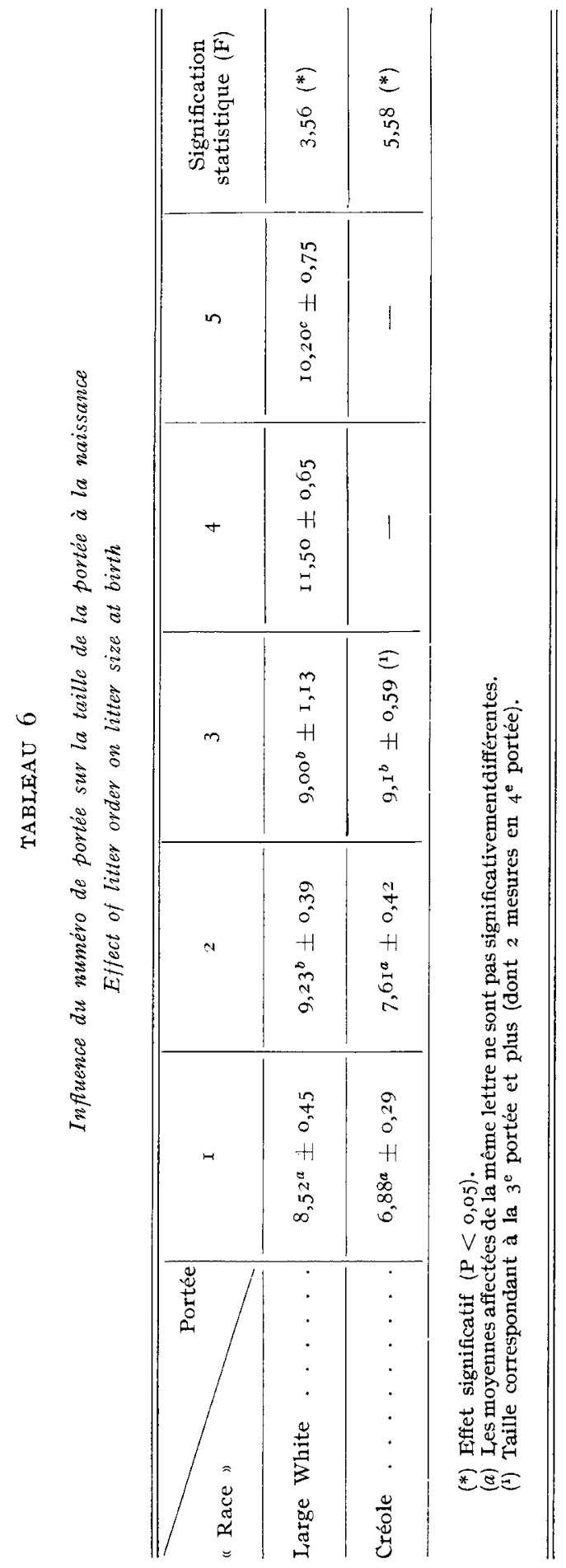




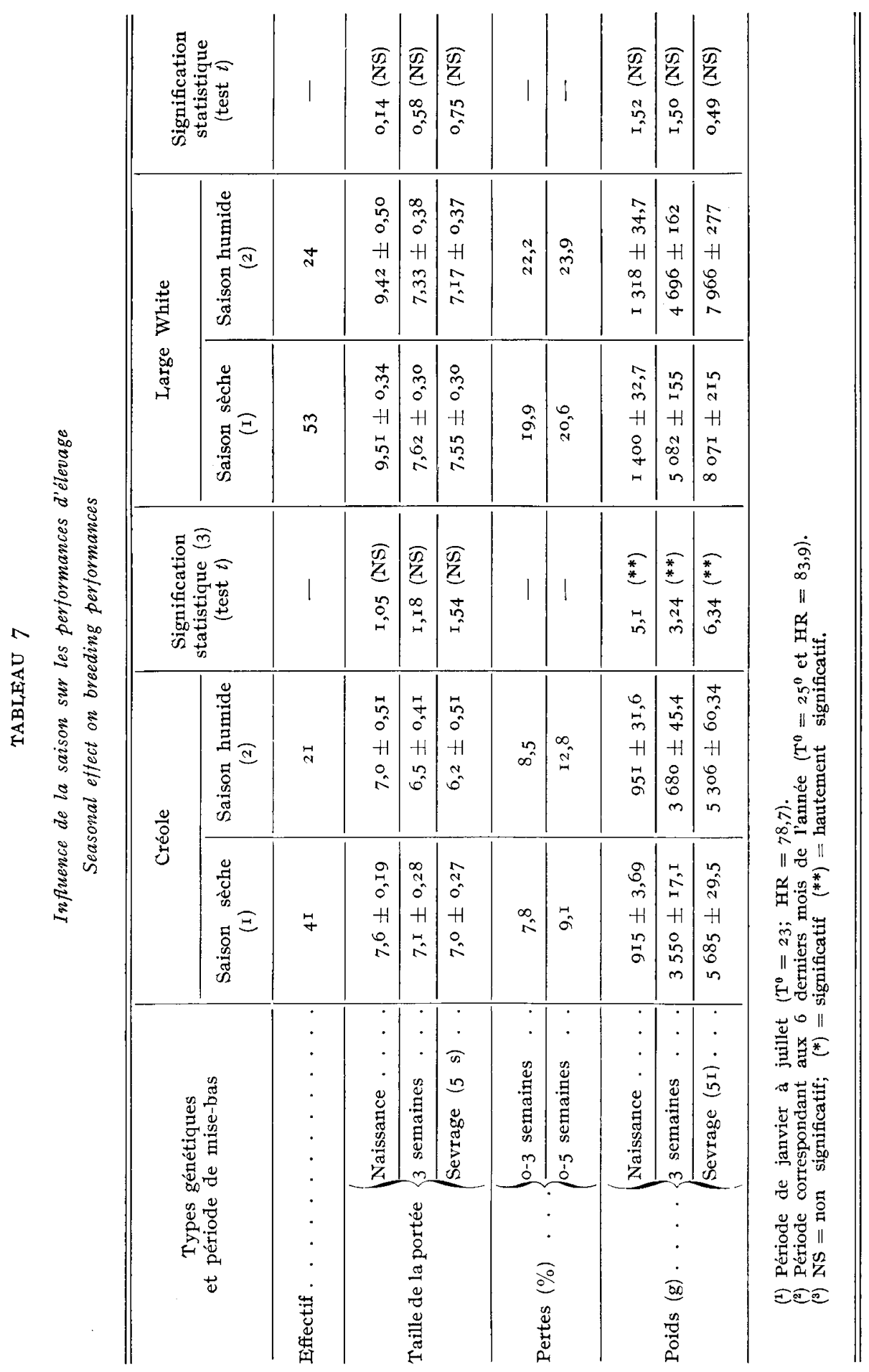




\section{IV. - Discussion}

La précocité sexuelle est une caractéristique intéressante qui n'est peut-être pas suffisament valorisée dans les élevages. Remarquons à ce propos que des informations précises sur l'âge et le poids à la puberté des races locales élevées dans leurs conditions naturelles sont rarement disponibles (DE ALRA, I972). Cependant, selon cet auteur les âges à la saillie fécondante des deux principales populations porcines du Brésil, le Piratinga (656 jours) et le Piau (560 jours), sont très élevés. Or, ces deux races nous semblent assez voisines du porc Créole quant à leur origine et leur aspect général.

Comme 1'ont montré DUÉE \& ÉTIENNE (I974) ces retards à la puberté pourraient s'expliquer par une sous-nutrition en cours de croissance et un déséquilibre alimentaire (SALMON-LEGaGneur I970, Dú́e et ÉtTEnNe I974), CunNinGHAM et al., I974).

- Les premières chaleurs des truies Large White se manifestent à un âge anormalement élevé (tabl. I) comparé aux valeurs moyennes (200 à 260 jours) des régions tempérées (CARREZ et al., I977, LEGAULT I973 et I978). Ces résultats concordent cependant avec ceux de SALMON-LEGAGNEUR (I970) et DuÉE et ÉTIENNE (I974) et les causes de retards à la puberté soulignées par MARTINAT et al. (1970) sont suffisamment nombreuses pour que la moyenne observée en Guadeloupe ne soit pas due à un effet d'échantillonnage.

En conclusion, dans les conditions particulières de notre troupeau expérimental l'avance de I04 jours à la puberté observée chez les truies Créoles semble attribuable pour l'essentiel à une différence d'origine génétique entre races.

L'intervalle sevrage-œstrus est relativement élevé dans les deux races (23 à 25 jours) comparativement aux résultats observés par ailleurs $(\mathbf{r} 8,7)$ et rapportés par FAнмy et al. (I979).

I1 en résulte un intervalle sevrage-fécondation de 36,2 pour les Large White et 28,5 pour les Créoles; les différences ne sont cependant pas significatives. Rappelons que pour cette même variable les estimations de LEGAULT (I975) et AUMAITRE et al (1976) sont comprises entre 20 et 25 jours.

Nous n'avons observé aucun effet significatif du numéro de portée sur la durée de la gestation contrairement aux résultats de OTMvedT I.T., STANISLAW C.M., Whatley J. A. (I965), Garnetti \& Rahnefeld (I979), Uzu (I979) et Aumaitre

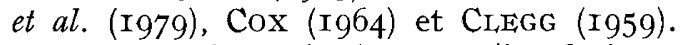

La première mise-bas a eu lieu 69 jours plus tôt chez les truies Créoles. Cette précocité a l'avantage d'améliorer très sensiblement la productivité numérique des truies par année de présence (LEGAulT I978).

Le gain de poids de la puberté à la fécondation est très faible chez la truie Créole (I,5 kg en $4 \mathrm{I}$ jours contre $3 \mathrm{~kg}$ en 8 jours en race Large White). On peut supposer que la présentation journalière et régulière d'un verrat non suivie de saillie en cas de chaleur entraîne une pertubation de la croissance en race Créole. Peut-être y aurait-il intérêt à faire saillir ces animaux dès la première chaleur ce qui réduirait d'un peu plus d'un mois l'âge à la première mise-bas.

Toutefois nous trouvons une corrélation significative de $+0,42$ entre 1'âge à la saillie fécondante et le nombre de porcelets nés : un optimum économique doit être recherché entre ces deux paramètres.

L'effet de la taille de la portée sur la croissance du porcelet est un résultat classique maintes fois confirmé par différents auteurs dont OmTVEDT (I965) et 
Aumaitre et al ig66). Mais dans tous les cas la supériorité du porc Large White est très nette : tout se passe comme si, au delà de 6 porcelets par portée, la truie Créole avait quelques difficultés à élever ses petits (poids au sevrage $4922 \mathrm{~g}$ contre 7780 g et 5600 contre 7330 dans les classes " 6 à 7 " et " 8 à Io " porcelets sevrés).

Le taux de survie relativement élevé des porcelets Créoles $(8,5 \mathrm{p}$. Ioo de perte de la naissance au sevrage) par rapport au Large White (2I,3 p. I0o) confirme les excellentes qualités maternelles de la truie Créole. Il en résulte que la taille de la portée au sevrage de ces deux races est assez voisine avec une productivité numérique de I3,8 et I4,6 porcelets respectivement pour les truies Créoles et Large White.

La variation de la taille de la portée (tab1. 6) en fonction du numéro de portée ne peut être précisée de manière formelle pour les Créoles, le troupeau étant relativement jeune. On peut cependant noter que pour les trois premières portées analysées, il y a une augmentation significative $(\mathrm{P}<0,05)$ du nombre de porcelets nés.

Les données enregistrées sur Large White sont conformes aux résultats classiques et sont en accord avec les travaux de KENNEDy et MoXLEy (I978), LEGAULT (I969), CANOPE et al (I973) sur 1'évolution de la taille de la portée en fonction du numéro de portée. Une augmentation significative est observée chez la truie Large White avec un maximum atteint dès la quatrième portée.

La nécessité de compléter les données classiques concernant les performances des animaux par une meilleure connaissance des effets de l'environnement a été maintes fois soulignée par différents auteurs (STEINBACH (I97I), LAWRENCE J. A. et al. (I970), Howarth (I969), Heitman et Hugues (I949), Frazer A. F. (I970) et FAHMY (I975)).

La faible taille de l'échantillon d'animaux dans chacune des races ne nous a pas permis de tester l'influence de la saison sur les performances des truies de même numéro de portées mais l'analyse globale (toutes portées confondues) nous fournit cependant quelques indications intéressantes.

En effet, si nous considérons les deux périodes allant de janvier à juillet (température : $23^{\circ}$, humidité relative $: 78,7$ ) et de août à décembre (température $25^{\circ}$, humidité relative : 83,9) (tab1. 7) on s'aperçoit que dans chacune des deux races dans leur ensemble, les meilleurs résultats sont obtenus au cours de la saison sèche. Notons que la variable la plus affectée pour les variations saisonnières est le pourcentage de mortalité de la naissance au sevrage.

\section{Conclusion}

Cette étude, situant le porc local par rapport aux introductions récentes fournit des éléments utiles à l'élaboration d'un programme d'amélioration de la production porcine en Guadeloupe.

En particulier, en dépit d'importantes différences de format et de comportement alimentaire, la truie Créole se révèle pratiquement aussi productive que la truie de race Large White en raison de sa rusticité et de son comportement maternel.

La définition d'un programme d'amélioration génétique pourrait être envisagée par la valorisation en croisement de la complémentarité qui existe entre ces deux populations : la précocité sexuelle, les qualités maternelles et la rusticité 
du porc Créole; la vitesse de croissance, la prolificité et la composition corporelle du porc Large White.

Toutefois cette recherche doit s'appuyer sur une analyse économique faisant intervenir notamment le coût de l'alimentation et les frais généraux (installations, main-d'œuvre). En effet si le porc Créole semble particulièrement bien adapté au climat tropical humide et à une nourriture locale pauvre en matière azotée, le porc Large White exige des installations mieux adaptées, un aliment plus coûteux et un surcroît de technicité.

Reçu pour publication en novembre 1980.

\section{Summary \\ Comparative studies of reproductive performances of Creole and Large White sows in Guadeloupe}

The swine population of the french west indies are represented by two main breeds : the Lavge White pigs and the local breed called "Creole " or "Planche " pigs.

From datas recorded in our experimental pig station we compared the reproductive characteristics of those two breeds.

Age and weight at puberty are $\mathrm{I} 7 \mathrm{I}, 4$ days and $52,3 \mathrm{~kg}$ for the local breed and 275 days and $107 \mathrm{~kg}$ for the Large White pig.

Other characteristics such as age and weight at the succesful mating, interval between weaning and the oestrus, interval between weaning and succesful mating, mean litter size at birth mean litter weight at birth, mean litter size at weaning, mean litter weight at weaning are respectively 212,9 days $53,8 \mathrm{~kg} ; 22,96$ days; 28,$47 ; 7,33 ; 906 \mathrm{~g} ; 6,71$ and 5322 for the "Creole " pigs on one hand and 283,$0 ; 110,0 ; 23,6 ; 36,2 ; 9,43 ; \mathrm{I} / 377 \mathrm{~g} ; 7,42 ; 8030 \mathrm{~g}$ for the Large White pig on the other hand. We also observed some influences of the season on the performance traits. In our climate conditions the mean litter size and mean litter weight seem to be higher from january to july when the weather is dry and less hot than is the raining period (from august to december). growth.

As far as the piglet growth is concerned the lower the litter size the better the piglet

We found for the two breeds reared in Guadeloupe French West Indies an annual productivity of 13,82 piglets/year/swine for the "Planche " pig and 14,6 for the Large White pigs.

\section{Références bibliographiques}

Aumaitre A., Legauli C., Salmon-Legagneur E., ig66. Aspects biométriques de la croissance pondérale du porcelet. Ann. Zootech., 15, 313.

AUmaitre A., Dagorn J., LEGault C., LE DEnmat M., I 976 . Influence of farm management and breed type on sow's conception weaning interval and productivity in France. Livest. Prod. Sci., 3, 75-83.

Aumaitre A., Deglaire B., Lebost S., i979. Prématurité de la mise bas chez la truie et signification du poids à la naissance du porcelet. Ann. Biol. anim. Biophys., 19, 267-275.

Canope I., LE Dividich J., Despors E., I973. Performances zootechniques de la Iarge-white aux Antilles. Bull. Tech. Prod. Anim., C.R.A.A.G. no 2-3, 86-94.

Carrez:S., Treil F., Dú́e P. H., Aumaitre A., i977. Influence sur la puberté de la truie de sa période de naissance et de sa durée d'allaitement. Ann. Zootech., 26, 62 I-625.

CLEGG M. T., I959. Factors affecting length and parturition in reproduction of domestic animals, vol. II (H. H. cole.and. PT cupps editors), Academic press, New York, p. 509.

Cox D: F., I964. Relation of litter size and other factors to the duration of gestation in the pig. J. Reprod. Fertil., 7, 405. 
Cunningham P. J., Naberc C. H., Zimmeran D. R., Peo E. R. Jr, i974. Influence of nutrition regime on age at puberty in gilts. $J$. Anim. Sci., 39, 63-67.

DE ALBA J., 1972. Productivité des races porcines indigènes et exotiques en Amérique latine. Rev. Mond. Zootech., 4, 25-28.

DUEE P., ÉTIENNE, 1974. Influence de l'alimentation pendant la croissance de la truie sur la maturité sexuelle et les performances de reproduction : résultats préliminaires. J. Rech. Porcine, 43-47.

DUTERTRE J. B., I667-r67r. Histoive des Antilles, 3 volumes, 4 tomes, Nouvelle réédition 1958. Société d'histoire de la Martinique, C.F.P. Fort-đe-France.

FAнMY M. H., I975. Effects of temperature and level of feeding during rearing on carcass and reproductive traits in pig. Livest. Prod. Sci., 2, 247-259.

Fahmy M. H., Holtmann W. B., BAKER R. D., I979. Failure to recycle after weaning and weaning to ostrus interval in crossbred sows. Anim. Prod., 29, 193-202.

Frazer A. F., 1970. Studies on heat stress in pigs in a tropical environment. Trop. Anim. Health Prod., 2, 76-87.

GARNETT I., RAHNEFEID G. W., 1979. Factors affecting gestation length in the pig. J. Anim. Sci., 59, 83-87.

HEITMAN H., HUGHes E. H., r949. The effects of air temperature and relative humidity on the physiological wellbeing of swine. J. Anim. Sci., I7I-I8I.

HowART B. Jr, I969. Fertility in ram following exposure to elevated ambient temperature and humidity. J. Reprod. Fert., 19, I 79.

KENNEDy B. W., MOXLEY J. E., 1978. Genetic and environmental factors influencing litter size sex-ratio and gestation length in the pig. Anim. Prod., 27, 35-42.

Laba't R. P., I722. Nouveau voyage aux Isles de 1'Amérique. Cavalier, Paris, 6 vol.

LAUVERgne J. J., CANOPE I., I979. Étude de quelques variants colorés du porc Créole de la Guadeloupe. Ann. Génét. Sél. Anim., 11, 381-39o.

Iawrence J. A., Turman E. J., Rica T., Shar A., Militer J. C., I970. A study of season effects in boar semen. OKLA Agric. Sta. Bull. M.P., 84, 77.

LEGAULT C., I969. Etude statistique et génétique des performances d'élevage de truie Largewhite, 1(3), $28 \mathrm{I}-298$.

I.EGAULT C., I973. Déterminisme génétique de la précocité sexuelle du taux d'ovulation et du nombre d'embryons chez la truie primipare : Héritabilité, effet d'hétérosis. $J$. Rech. Porcine, I 47 -I 54 .

LEGaulT C., I978. Génétique et reproduction chez le porc. J. Rech. Porcine.

LEGAULT C., I978. Contribution du contrôle des portées dans les élevages à l'amélioration génétique de la productivité des truies. $29^{\mathrm{e}}$ Meeting annuel de la F.E.Z., 5-7/6/78.

IEGAUlT C., AUMAITRE A., DUMESNIL DU BUISSON F., 1975. The improvement of sow productivity a review of recent experiments in France. Livest. Prod. Sci., 2, 23.5-246.

LEMENTEC J. C., x970. Étude monographique du porc Créole en Guadeloupe, B.T.I., 25I, 436-446.

Martinat F., Legault C., Dumesnil du Buisson F., Ollivier L., Signoret J. P., i97o. Étude des retards de puberté chez la truie. J. Rech. Porcine, 47-53.

OMTVEDT I. T., STANislaw C. M., Whatrey J. A., I965. Relationship of gestation length age and weigth at breeding and gestation to sow productivity at farrowing. J. Anim. Sci., 24, $52 \mathrm{I}$.

STEINBACH J., I97I. Effects of season and breed on sow performance in the seasonal equatorial climate of southern Nigeria. Agric. Sci. Camb., 77, 331-336.

Salmon-LEGagneur E., I970. Étude de quelques variations de l'âge et du poids des truies Largewhite au $\mathrm{I}^{\mathrm{er}}$ ostrus. $J$. Rech. Porcine, 4I-46.

UZU G., I979. Influence de la saison et du verrat sur les principaux paramètres de la productivité et sur la durée de gestation. Ann. Zootech., 28, 31 5-323. 